\title{
Theoretical Insight into the Role of Defects and Facets in the Selectivity of Products in Water Oxidation over Bismuth Vanadate $\left(\mathrm{BiVO}_{4}\right)$
}

\author{
Supporting information \\ Taifeng Liu*, Ruyue Liu, Qiuye Li, and Jianjun Yang \\ National \& Local Joint Engineering Research Center for Applied Technology of \\ Hybrid Nanomaterials, Henan University, Kaifeng, 475004, China. \\ Corresponding author: tfliu@vip.henu.edu.cn
}

Number of pages: 5

Number of Figures: 3

Number of Tables: 1 


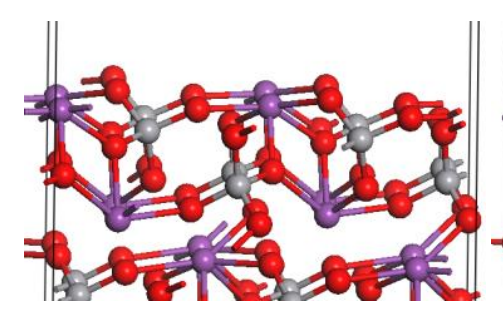

101

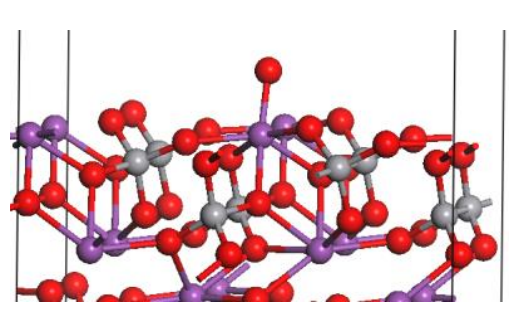

101-02

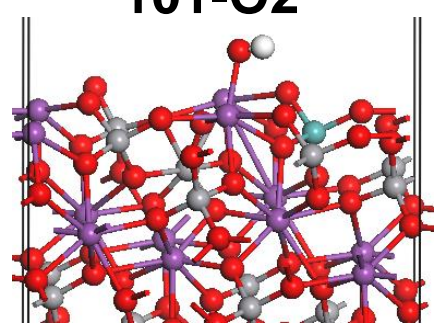

101-Mo-OH

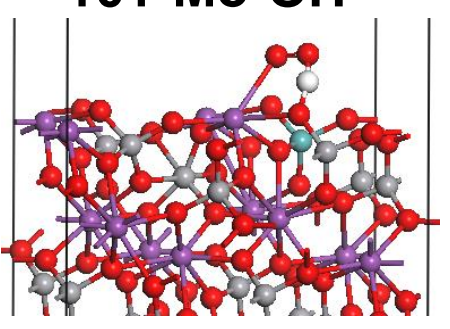

101-Mo-OOH

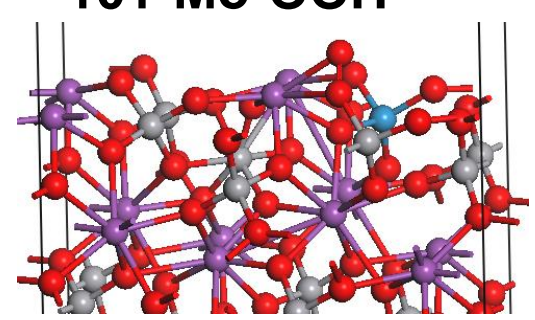

101-W-O1

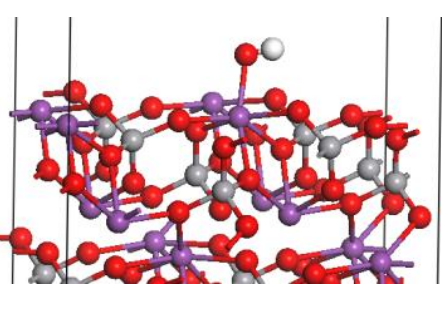

101-OH

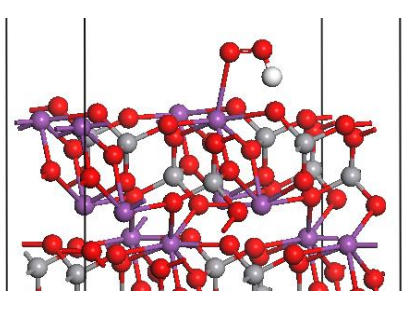

101-OOH

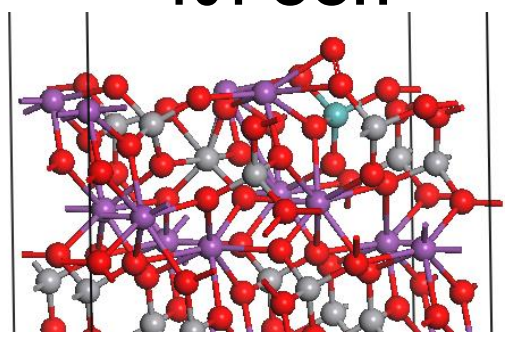

101-Mo-01

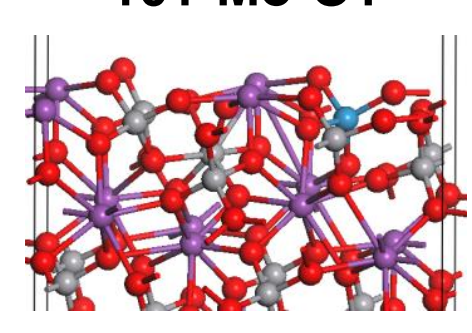

101-W

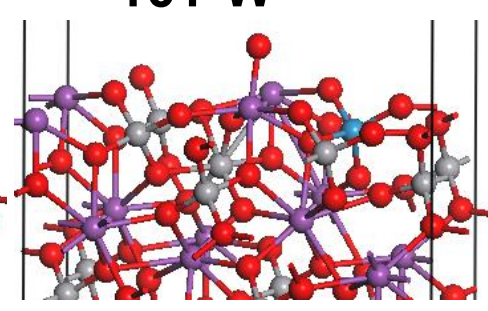

101-W-O2

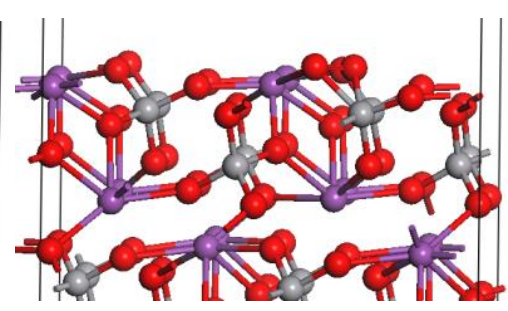

101-01

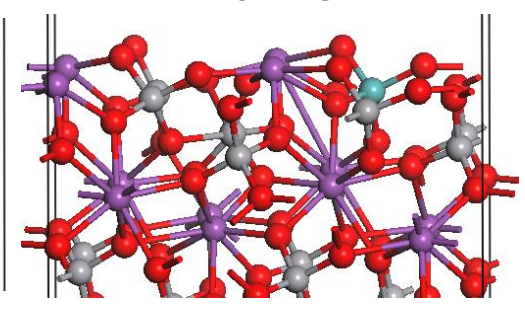

101-Mo

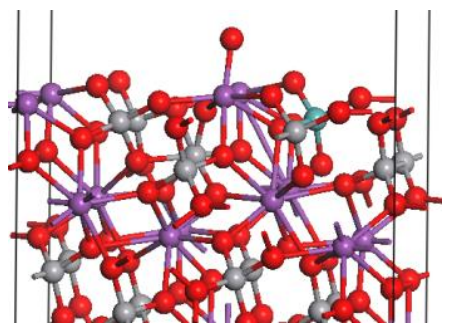

101-Mo-O2

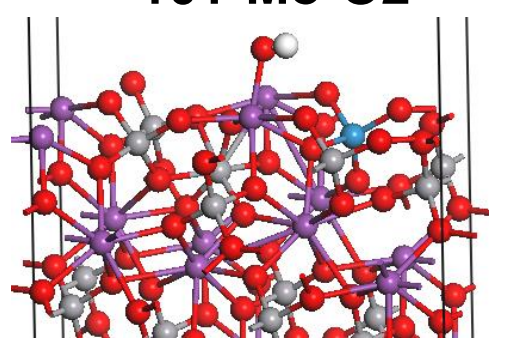

$101-\mathrm{W}-\mathrm{OH}$

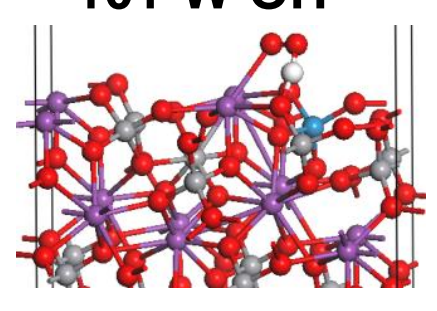

101-W-OOH

Figure S1: The structures of OER intermediates *, $\mathrm{OH}^{*}, \mathrm{O}^{*}, \mathrm{O}^{*}$, and $\mathrm{OOH}^{*}$ on clean (101) and Mo/W doping surfaces. 


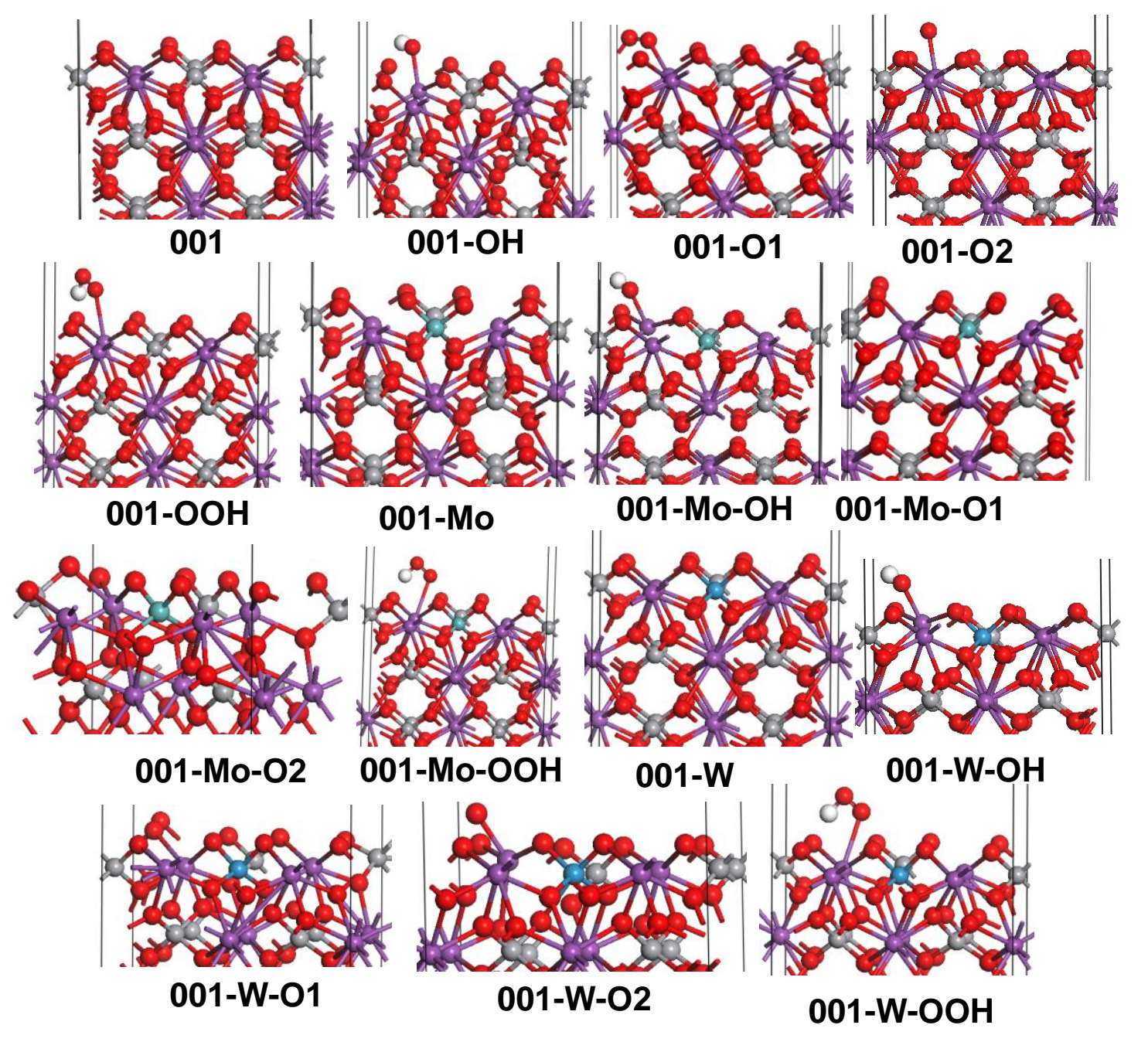

Figure S2: The structures of OER intermediates *, $\mathrm{OH}^{*}, \mathrm{O}^{*}, \mathrm{O}^{*}$, and $\mathrm{OOH}^{*}$ on clean (001) and Mo/W doping surfaces. 

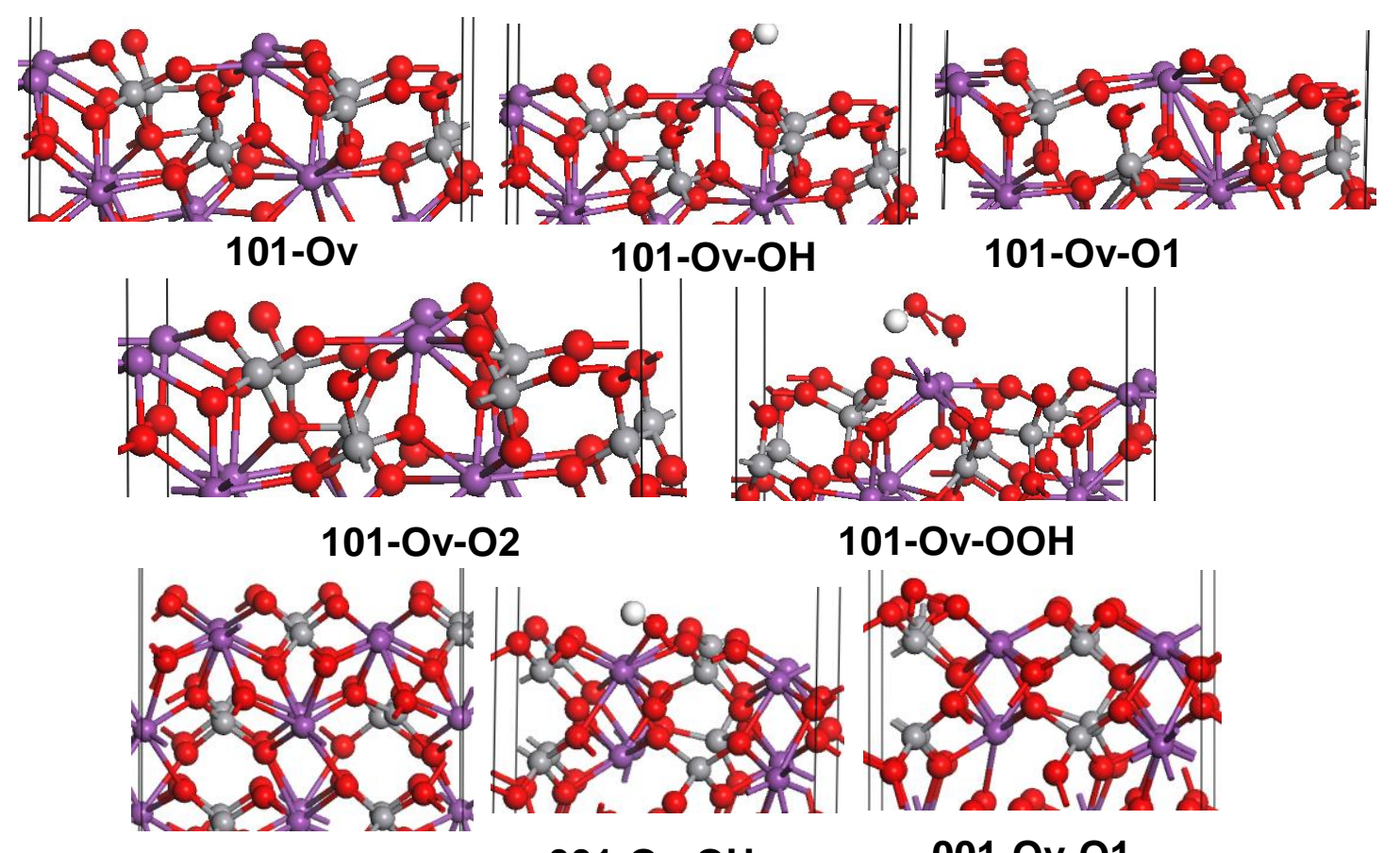

101-Ov-OOH

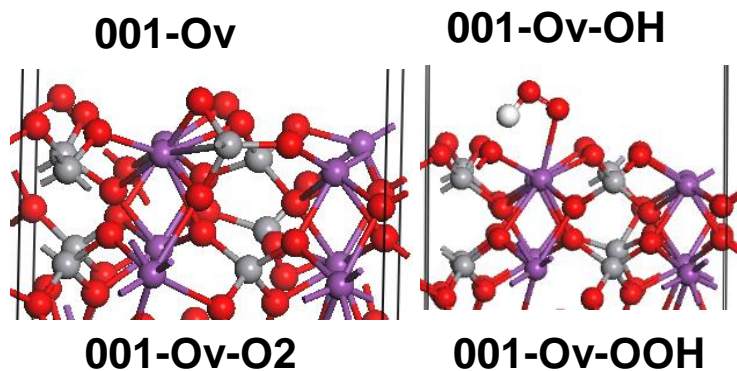

Figure S3: The structures of OER intermediates *, $\mathrm{OH}^{*}, \mathrm{O}^{*}, \mathrm{O}^{*}$, and $\mathrm{OOH}^{*}$ on clean (001) and (101) surfaces in the present of oxygen vacancy. 
Table S1: The free energies and over-potentials $(\eta)$ for two-electron and four electron water oxidation on all the surfaces

\begin{tabular}{|c|c|c|c|c|c|c|}
\hline surfaces & $\Delta G_{\mathrm{OH}}(\mathrm{eV})$ & $\Delta G_{\mathrm{O}}(\mathrm{eV})$ & $\Delta G_{\mathrm{OOH}}(\mathrm{eV})$ & Products & $4 e-\eta^{O E R}(\mathrm{~V})$ & $2 e-\eta^{O E R}(\mathrm{~V})$ \\
\hline \multirow[t]{2}{*}{101} & 2.25 & 3.22 & 4.64 & $\mathrm{O} 2$ & 1.02 & 0.49 \\
\hline & 2.25 & 4.41 & 4.63 & $\mathrm{H} 2 \mathrm{O} 2$ & 1.02 & 0.49 \\
\hline \multirow[t]{2}{*}{$101-\mathrm{Ov}$} & 0.95 & 2.61 & 4.36 & $\mathrm{O} 2$ & 0.52 & -- \\
\hline & 0.95 & 3.33 & 4.36 & $\mathrm{O} 2$ & 1.15 & 0.62 \\
\hline \multirow[t]{2}{*}{ 101-Mo } & 2.18 & 3.98 & 4.56 & $\mathrm{H} 2 \mathrm{O} 2$ & 0.95 & 0.42 \\
\hline & 2.18 & 4.41 & 4.56 & $\mathrm{H} 2 \mathrm{O} 2$ & 1.00 & 0.47 \\
\hline \multirow[t]{2}{*}{$101-W$} & 2.19 & 3.98 & 4.57 & $\mathrm{H} 2 \mathrm{O} 2$ & 0.96 & 0.43 \\
\hline & 2.19 & 4.43 & 4.57 & $\mathrm{H} 2 \mathrm{O} 2$ & 1.01 & 0.48 \\
\hline \multirow[t]{2}{*}{001} & 2.57 & 3.82 & 4.70 & $\begin{array}{r}\mathrm{OH} \\
\text { radical }\end{array}$ & 1.34 & 0.81 \\
\hline & 2.57 & 5.08 & 4.69 & $\begin{array}{r}\mathrm{OH} \\
\text { radical }\end{array}$ & 1.34 & 0.81 \\
\hline \multirow[t]{2}{*}{$001-\mathrm{Ov}$} & 0.96 & 3.21 & 5.23 & $\mathrm{O} 2$ & 1.02 & 0.49 \\
\hline & 0.96 & 1.71 & 5.23 & $\mathrm{O} 2$ & 2.34 & -- \\
\hline \multirow[t]{2}{*}{ 001-Mo } & 1.50 & 3.26 & 4.74 & $\mathrm{O} 2$ & 0.53 & 0 \\
\hline & 1.50 & 3.52 & 4.75 & $\mathrm{H} 2 \mathrm{O} 2$ & 0.79 & 0.26 \\
\hline \multirow[t]{2}{*}{$001-\mathrm{W}$} & 1.44 & 3.22 & 4.72 & $\mathrm{O} 2$ & 0.55 & 0.02 \\
\hline & 1.44 & 4.17 & 4.72 & $\mathrm{H} 2 \mathrm{O} 2$ & 1.50 & 0.32 \\
\hline
\end{tabular}

